\title{
Use of motion peculiarities of test particles for estimating degree of coherence of optical fields
}

\author{
Zenkova C. Yu., Gorsky M. P., Soltys I. V. and Angelsky P. O. \\ Chernivtsi National University, 2 Kotsyubynsky St., Chernivtsi, Ukraine, \\ e-mail: zenkova@itf.cv.ua
}

Received: 26.07 .2012

\begin{abstract}
We discuss interconnections between the depth of spatial inhomogeneity of energy distribution of the optical fields and the velocity of motion of test particles for the cases of different light scattering mechanisms during interaction with light. We suggest an additional tool for determining the degree of coherence of superposing waves that propagate along mutually orthogonal directions and have orthogonal polarisations, being linearly polarised in the incidence plane. The use of velocity of the test particles while estimating the degree of coherence of optical fields is suggested for the first time.
\end{abstract}

Keywords: degree of coherence, nanoparticles, Poynting vector, light scattering

PACS: $42.25 . \mathrm{Kb}$

UDC: 535.41

\section{Introduction}

One of the most urgent tasks of the present-day biomedicine is controlling microparticles and nanoparticles of different natures with the aid of laser radiation. The objects under control may be a colloid particle, a molecule, an atom, a cell, or include biological objects (e.g., a DNA molecule or an organelle cell). It is also tempting to assume that the methods for diagnosing pathologies of biological tissues, which employ optical methods developed in the work [1], can be greatly expanded using the techniques for controlling nanoobjects and microoobjects.

One readily understands that technologies of particle manipulation represent a powerful tool while working with microobjects of different natures. The process of trapping and controlling particles depends on characteristics of laser beams. At present, a number of techniques relying on optical beams of various types (Gaussian [2,3] and bottle [4] beams, zero-order Bessel beams [5], self-focused laser beams [6], and even evanescent fields [7, 8]) have been explored. One of the possibilities for controlling micro- and nanoparticles is to use dislocations of wave fronts resulting, e.g., from interference among the beams with simple wave fronts [9].

A principal prerequisite of efficient particle manipulation using the laser beams is their complete coherence. In practice, however, any laser field is always coherent only partially [10]. Moreover, a possibility for highly accurate focusing, and then producing a force component capable to change direction of motion of an object, is determined by the degree of coherence of the beams [11-12]. For the light beams with high coherence, the optical force may be used to trap a particle, while the optical force appearing for the light beams with intermediate coherence may be employed for guiding and accelerating that particle [13].

The aim of this work is to investigate resources contained in the information about the motion dynamics for nanosize- and microsize particles, when determining the degree of coherence 
of the two superposing mutually orthogonal waves, which propagate along mutually orthogonal directions and are linearly polarised in the incidence plane.

\section{Statement of the problem}

At present, optical traps and micromanipulators are created taking into consideration the basic properties of laser radiation and the condition that different components of the optical force (e. g., the components of radiation pressure, the scattering force, and the gradient force [14]) compete with each other. The scattering force is proportional to the radiation intensity and acts along the direction of the incident laser beam. The gradient component acts in the direction of the gradient intensity. These components can create a stable point, which is localised near the focus. If one of the components is larger or smaller than the other, an equilibrium point is absent and a directional motion of a particle is observed. The availability of field pressure, which is associated with internal angular momentum of the field, results in arising of some energy flux, because the momentum density and the density of the energy flux are interconnected [15]. The momentum of the field passed on to a particle, causes a movement of that particle.

Calculations of the optical force affecting particles in cases of different light scattering mechanisms depend on the sizes and properties of those test particles, as well as on the properties and characteristics of the optical field wherein the particles are localised. As a rule, the following groups of particles should be distinguished.

1. The particles whose sizes are much smaller than the light wavelength or, according to more accurate definition, those satisfying the condition $r<</ 20$ (with $r$ and $\lambda$ denoting respectively the radius of the particle and the light wavelength). These particles are called as Rayleigh particles in the literature [16]. Their interaction with electromagnetic waves is described in the framework of Rayleigh light scattering mechanism. In this case an approximation of point dipoles may be introduced.

2. The particles with the sizes of the order of $\lambda / 5$. The physical mechanism [16] that determines interaction of the particles with the optical field is explained in the context of Rayleigh light scattering approximation, which agrees with a so-called Mie theory. Considerations of each component of the optical force and calculations of the gradient, scattering and the absorbing components are typical for the particles of this type.

3. The particles with the sizes of the order of $\lambda$ [17]. To describe interaction of these particles with the optical field, a generalised Lorenz-Mie theory is applied.

4. The particles whose sizes are $r>>\lambda$ [17]. Then the approximation of geometrical optics is used, based upon the belief that the ray transfers a linear momentum. A transfer of that momentum from the ray to the particle causes the gradient and scattering components of the optical force.

It is well known that the instantaneous value $\mathbf{S}$ of the Poynting vector is determined by the relation $\mathbf{S}=\mathbf{E} \times \mathbf{H}$, where as usual $\mathbf{E}$ and $\mathbf{H}$ mean the electric and magnetic field strength vectors, respectively. The density of the energy current is $S=E H=\frac{w}{\sqrt{\mu \varepsilon \mu_{0} \varepsilon_{0}}}$, where $w$ stands for the electromagnetic field energy, $\varepsilon$ and $\mu$ are respectively the dielectric permittivity and the magnetic permeability, and $\varepsilon_{0}$ and $\mu_{0}$ denote respectively the vacuum permittivity and the vacuum permeability. It is known that the relation $w=2 w_{E}=2 w_{H}$ holds true, where the energy 
densities $w_{E}$ and $w_{H}$ of the electric and magnetic fields are $w_{E}=\frac{1}{2} \varepsilon \varepsilon_{0}(\mathbf{E}, \mathbf{E})$ and $w_{H}=\frac{1}{2} \mu \mu_{0}(\mathbf{H}, \mathbf{H})$, respectively. The average Poynting vector represents a time-averaged distribution of the relevant instantaneous values, with averaging over the whole oscillation period.

Let us consider a superposition of two plane waves W1 and W2 of equal amplitudes, which are polarised in the plane of incidence (see Fig. 1). These waves would determine the averaged electric (magnetic) energy density and the normal propagation direction of the resulting wave [18, 25].

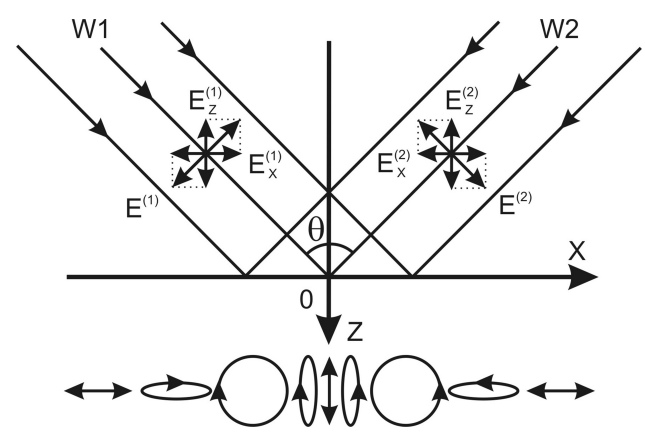

Fig. 1. Superposition of plane waves which have equal amplitudes and are linearly polarised in the incidence plane in case of interference angle of $90^{\circ}$. A periodic spatial modulation of polarization [19-21] takes place in the plane of incidence.

Let $\mathbf{E}^{(1)}\left(\mathbf{r}_{1}, t\right) \quad\left(\mathbf{H}^{(1)}\left(\mathbf{r}_{1}, t\right)\right)$ and $\mathbf{E}^{(2)}\left(\mathbf{r}_{2}, t\right) \quad\left(\mathbf{H}^{(2)}\left(\mathbf{r}_{2}, t\right)\right)$ be the electric (magnetic) field vectors for the first and second interacting waves, respectively. The resulting electric (magnetic) field vector in the detection plane at the point $\mathbf{r}$ is defined as $\mathbf{E}(\mathbf{r})=\mathbf{E}^{(1)}(\mathbf{r})+\mathbf{E}^{(2)}(\mathbf{r})$ $\left(\mathbf{H}(\mathbf{r})=\mathbf{H}^{(1)}(\mathbf{r})+\mathbf{H}^{(2)}(\mathbf{r})\right)$. We will find the direction of the Poynting vector for the resulting wave as $\mathbf{S}=\mathbf{S}^{(1)}+\mathbf{S}^{(2)}+\mathbf{E}^{(1)} \times \mathbf{H}^{(2)}+\mathbf{E}^{(2)} \times \mathbf{H}^{(1)}$, where $\mathbf{S}^{(1)},\left(\mathbf{S}^{(2)}\right)$ are the Poynting vectors of the superposing waves. Since the waves W1 and W2 are propagate along mutually orthogonal directions in the interference scheme suggested by us, the direction of the resulting vector $\mathbf{S}$ is determined by the direction of the vector obtained when summing over the Poynting vectors $\mathbf{S}^{(1)}$ and $\mathbf{S}^{(2)}$ of the initial superposing waves.

In order to find the distribution of the volume energy density, we use a matrix approach and define the mutual coherence matrix describing the field correlation at two different spatial points $\mathbf{r}_{1}$ and $\mathbf{r}_{2}$ [22, 23]. It is given by $W\left(\mathbf{r}_{1}, \mathbf{r}_{2}, t\right)=<E_{i}^{(1)}\left(\mathbf{r}_{1}, t\right) E_{j}^{(2)^{*}}\left(\mathbf{r}_{2}, t\right)>$, where $i, j=x$, $z$. In this approximation, the components of degree of mutual coherence of the fields are expressed as

$$
\eta_{i j}\left(\mathbf{r}_{1}, \mathbf{r}_{2}, \tau\right)=\frac{W_{i j}\left(\mathbf{r}_{1}, \mathbf{r}_{2}, \tau\right)}{\sqrt{\operatorname{tr}\left[W\left(\mathbf{r}_{1}, \mathbf{r}_{1}, 0\right)\right]} \cdot \sqrt{\operatorname{tr}\left[W\left(\mathbf{r}_{2}, \mathbf{r}_{2}, 0\right)\right]}}=\frac{W_{i j}\left(\mathbf{r}_{1}, \mathbf{r}_{2}, \tau\right)}{\sqrt{\sum_{i j} W_{i i}\left(\mathbf{r}_{1}, \mathbf{r}_{1}, 0\right) W_{j j}\left(\mathbf{r}_{2}, \mathbf{r}_{2}, 0\right)}} .
$$

Let us assume that $\tau=0$. This enables estimating the spatial coherence of the optical field.

Now we define the time-averaged density of the energy current at the point of observation $\mathbf{r}$ (see [24]):

Ukr. J. Phys. Opt. 2012, V13, №4 


$$
S=\sqrt{\frac{\varepsilon \varepsilon_{0}}{\mu \mu_{0}}} \sum_{i, j}\left\{\phi_{i j}^{(1)}(\mathbf{r})+\phi_{i j}^{(2)}(\mathbf{r})+2 \sqrt{\operatorname{tr}\left[W\left(\mathbf{r}_{1}, \mathbf{r}_{1}, 0\right)\right] \operatorname{tr}\left[W\left(\mathbf{r}_{2}, \mathbf{r}_{2}, 0\right)\right]}\left|\eta_{i j}^{(1,2)}\right| \cos \left[\alpha_{i j}^{(1,2)}\right] \cos \left[\delta_{e}\right]\right\},
$$

where $\varphi_{i j}^{(m)}(\mathbf{r})=<E_{i}^{(m)}(\mathbf{r}, t) E_{j}^{*(m)}(\mathbf{r}, t)>, m=1,2, i, j=x, z, \alpha_{i j}^{(1,2)}$ is an argument of $\eta_{i j}^{(1,2)}$ and determines the phase difference between the components $i$ and $j$ of the field, and $\delta_{e}$ denotes the phase difference of the beams (here their electrical parts) at the detection plane.

According to Eq. (2), spatial distribution of the time-averaged energy current density determines the current at different points of the detection plane. It is unambiguously determined by the degree of coherence of the superposing waves $\eta^{(1,2)}$. The direction of the resulting energy current is set by the directions of the Poynting vectors of these waves.

When accounting for superposition of our mutually orthogonal linearly polarised fields, a modulation of the averaged Poynting vector magnitude, i.e. a spatial modulation of the energy density, is noticed in the observation plane. The optical force associated with the Poynting vector forms some distribution of particles in this plane. Moreover, any change in the degree of coherence would lead to changing modulation depth of the volume energy density. In its turn, this would determine the optical force and so the peculiarities of motion of the particle.

As stated before, the forces resulting from the laser beam and influencing the particles depend in general on the properties of both the beam and those particles. Further on, we will consider the behaviour of test particles in an inhomogeneous optical field. According to the experiment suggested in the study [25], these are the particles peculiar for the golden hydrosol (of about $1 \mathrm{~nm}$ and $100 \mathrm{~nm}$ in size; considered at the room temperature). The mechanical influence of spin flows $[26,27]$ on the motion of these particles is not dealt with hereafter. Let us analyse formation of the optical force $\mathbf{F}_{o p t}$ that affects the particles.

The direction of gradient component of the optical force is related to spatial distribution gradient of the volume energy density. The directions of the scattering and the absorbing components are given by the direction of the energy flow propagation. At the same time, the direction of the reflecting component depends on orientation of the particles' surfaces.

Basing on the polarisability $\alpha=\alpha^{\prime}+i \alpha^{\prime \prime}$ of the molecule, one can calculate the gradient component of the optical force as $\mathbf{F}_{\text {grad }}=-\frac{1}{2} n \alpha^{\prime} \nabla E^{2}$ (with $n$ being the refractive index of a surrounding medium). The molecular polarisability $\alpha$ can be derived from the Clausius-Mossoti equation ( $\alpha=3 V \frac{\varepsilon_{p}-\varepsilon_{m}}{\varepsilon_{p}+2 \varepsilon_{m}}$, with $\varepsilon_{p}$ and $\varepsilon_{m}$ being the dielectric permittivities of the gold particle and the surrounding medium, respectively). For the golden particles in water we have $n=0.32+2.65 i$ [28], where both the real and imaginary parts of the dielectric permittivity are be calculated using the Kramers-Kronig relations.

It is known that $\mathbf{S}=\frac{c}{4 \pi} \sqrt{\frac{\varepsilon}{\mu}} E^{2} \mathbf{S}$, where $\mathbf{S}$ is the unit vector of the propagation direction [29] and $S=\frac{c}{4 \pi} \sqrt{\frac{\varepsilon}{\mu}} E^{2}$. Then we obtain $E^{2}=\frac{4 \pi S}{c} \sqrt{\frac{\mu}{\varepsilon}}$ and so 


$$
\mathbf{F}_{\text {grad }}=-\frac{1}{2} n \alpha^{\prime} \nabla E^{2}=-\frac{2 \pi n \alpha^{\prime}}{c} \sqrt{\frac{\mu}{\varepsilon}} \nabla S .
$$

Thus, one can easily find the field gradient for the general case. The gradient of an arbitrary function can be calculated at any point of the plane, including the observation plane. It is also known that the modulus of the gradient of any function is equal to the greatest rate of changes of that function at the chosen point. Therefore it is feasible to calculate the gradient distribution of the volume energy density as $\nabla S=\vec{i} \frac{\partial S}{\partial x}+\vec{k} \frac{\partial S}{\partial z}$, assuming that a distribution of the energy density takes place in the plane $x z$.

Let the coordinates of the point in the region of the minimal volume energy density be $x_{\min }$ and $z_{\min }$. In order to define the gradient $\nabla S$ at the arbitrary point $m$ with the coordinates $x_{m}$ and $z_{m}$ in the observation plane, the remoteness of the given point relative to the nearest point is marked off in the minimal energy region. We preset some shift of the coordinates of the given point with respect to the coordinates of the point inside the minimal energy region. Then one can write the following expression for the point $m$ :

$$
|\nabla S|_{m}=\sqrt{\left(\frac{\partial S_{m}}{\partial x_{m}}\right)^{2}+\left(\frac{\partial S_{m}}{\partial z_{m}}\right)^{2}}=\sqrt{\left(\frac{\Delta S_{m}}{\Delta x_{m}}\right)^{2}+\left(\frac{\Delta S_{m}}{\Delta z_{m}}\right)^{2}}=\Delta S_{m} \sqrt{\frac{1}{\left(\Delta x_{m}\right)^{2}}+\frac{1}{\left(\Delta z_{m}\right)^{2}}}
$$

where $\Delta x_{m}=x_{\text {min }}-x_{m}, \Delta z_{m}=z_{\text {min }}-z_{m}, \Delta S_{m}=S_{\text {min }}-S_{m}$, and

$$
\begin{aligned}
& S_{\min }=\sqrt{\frac{\varepsilon \varepsilon_{0}}{\mu \mu_{0}}} \sum_{i, j}\left\{\phi_{i j}^{(1)}(\mathbf{r})+\phi_{i j}^{(2)}(\mathbf{r})-2 \sqrt{\operatorname{tr}\left[W\left(\mathbf{r}_{1}, \mathbf{r}_{1}, 0\right)\right] \operatorname{tr}\left[W\left(\mathbf{r}_{2}, \mathbf{r}_{2}, 0\right)\right]} \cdot \eta_{i j}^{(1,2)}\right\}, \\
& S_{m}=\sqrt{\frac{\varepsilon \varepsilon_{0}}{\mu \mu_{0}}} \sum_{i, j}\left\{\phi_{i j}^{(1)}(\mathbf{r})+\phi_{i j}^{(2)}(\mathbf{r})+2 \sqrt{\operatorname{tr}\left[W\left(\mathbf{r}_{1}, \mathbf{r}_{1}, 0\right)\right] \operatorname{tr}\left[W\left(\mathbf{r}_{2}, \mathbf{r}_{2}, 0\right)\right]} \eta_{i j}^{(1,2)} \cos \left[\left(\delta_{e}\right)_{m}\right]\right\} .
\end{aligned}
$$

As a result, one gets

$$
\Delta S_{m}=-2 \sqrt{\frac{\varepsilon \varepsilon_{0}}{\mu \mu_{0}}} \sum_{i j} \sqrt{\operatorname{tr}\left[W\left(\mathbf{r}_{1}, \mathbf{r}_{1}, 0\right)\right] \operatorname{tr}\left[W\left(\mathbf{r}_{2}, \mathbf{r}_{2}, 0\right)\right]} \eta_{i j}^{(1,2)}\left(1-\cos \left[\left(\delta_{e}\right)_{m}\right]\right)=\sqrt{\frac{\varepsilon}{\mu}} K_{m} \sum_{i j} \eta_{i j}^{(1,2)} .
$$

Here $K_{m}=-2 \sqrt{\frac{\varepsilon_{0}}{\mu_{0}}} \sqrt{\operatorname{tr}\left[W\left(\mathbf{r}_{1}, \mathbf{r}_{1}, 0\right)\right] \operatorname{tr}\left[W\left(\mathbf{r}_{2}, \mathbf{r}_{2}, 0\right)\right]}\left(1-\cos \left[\left(\delta_{e}\right)_{m}\right]\right)$ represents the parameter that characterisers the energy density for the chosen point $m$ of the optical field. It depends on the phase difference between the initial waves at this point. Let us write out the main result:

$$
\Delta S_{m}=\sqrt{\frac{\varepsilon}{\mu}} K_{m} \eta^{(1,2)}
$$

Following from this expression, one can represent the gradient component of the optical force as

$$
\left(F_{\text {grad }}\right)_{m}=-\frac{2 \pi n \alpha^{\prime}}{c} K_{m} \eta^{(1,2)} \sqrt{\frac{1}{\left(\Delta x_{m}\right)^{2}}+\frac{1}{\left(\Delta z_{m}\right)^{2}}} .
$$

The effect of the gradient force is linked to the degree of coherence of the interacting fields. 
According to the results [16], one can define the scattering and the absorbing components of the optical force in the frame of the Rayleigh approximation as

$$
\begin{gathered}
\mathbf{F}_{s c a t}=\frac{n}{c} C_{s c a t} \mathbf{S}, C_{s c a t}=\frac{k^{4}}{6 \pi \varepsilon_{0}^{2}}|\alpha|^{2}, k=2 \pi / \lambda, \\
\mathbf{F}_{a b s}=\frac{n}{c} C_{a b s} \mathbf{S}, C_{a b s}=\frac{k}{\varepsilon_{0}}\left|\alpha^{\prime \prime}\right|^{2} .
\end{gathered}
$$

Thus, we obtain

$$
\mathbf{F}_{\text {opt }}=\mathbf{F}_{\text {grad }}+\mathbf{F}_{\text {scat }}+\mathbf{F}_{a b s}
$$

Depending on the relationships among the gradient, scattering or the absorbing components of the optical force, the particles are either trapped by the field or they move in the direction defined by the energy flow.

Suppose that the particles under analysis are situated in a viscous medium. Let us also assume that a mixture consists of a viscous incompressible carrier phase (see [30]) and spherical particles with the radius $r$ and the mass $M$. When moving in the liquid, the particles experience a friction force. If the velocity $\mathbf{v}$ remains constant, the force is determined by the Stokes law $\left(\mathbf{F}_{s t}=B \mathbf{v}=6 \pi \eta r \mathbf{v}\right.$, where $B$ means the friction coefficient for the particle, $\eta$ the viscosity of the dispersive medium). Then the equation of motion of the particle under the optical force in the viscous medium will be as follows:

$$
M \frac{d \mathbf{v}}{d t}=\mathbf{F}_{o p t}+\mathbf{F}_{s t} .
$$

In the first approximation we ignore the Brownian motion of the particles and suppose them to be motionless until being brought into the optical field.

Let us consider the particle $m$ with the mass $M$ and the radius $r$, which is located at the field point with the coordinates $x_{m}$ and $z_{m}$. When solving the differential equation of motion written in the scalar form,

$$
M \frac{d v_{m}}{d t}=-6 \pi \eta r v-\frac{2 \pi n}{c} \alpha^{\prime} K_{m} \eta^{(1,2)} \sqrt{\frac{1}{\left(\Delta x_{m}\right)^{2}}+\frac{1}{\left(\Delta z_{m}\right)^{2}}}+\left\{C_{a b s}+C_{s c a t t}\right\} \frac{n}{c} S_{m},
$$

we obtain

$$
\begin{aligned}
& v_{m}(t)=\frac{1}{M}\left(e^{-\frac{6 \pi \eta r}{M} t}+1\right) \times\left\{\frac{2 \pi n}{c} \alpha^{\prime} K_{m} \eta^{(1,2)} \sqrt{\frac{1}{\left(\Delta x_{m}\right)^{2}}+\frac{1}{\left(\Delta z_{m}\right)^{2}}}\right. \\
& \left.-\left\{C_{a b s}+C_{\text {scatt }}\right\} \frac{n}{c} \sqrt{\frac{\varepsilon \varepsilon_{0}}{\mu \mu_{0}}} \sum_{i, j}\left\{\phi_{i j}^{(1)}(\mathbf{r})+\phi_{i j}^{(2)}(\mathbf{r})+2 \sqrt{\operatorname{tr}\left[W\left(\mathbf{r}_{1}, \mathbf{r}_{1}, 0\right)\right] \operatorname{tr}\left[W\left(\mathbf{r}_{2}, \mathbf{r}_{2}, 0\right)\right]} \eta_{i j}^{(1,2)} \cos \left[\left(\delta_{e}\right)_{m}\right]\right\}\right\}
\end{aligned}
$$

As seen from this formula, the velocity $v_{m}(t)$ of each separate particle depends upon its position in the optical field.

Now we perform averaging over the ensemble of particles localised in the optical field. The velocity of motion of $m$ particles averaged over their ensemble may be written as 


$$
\begin{aligned}
& \bar{v}(t)=\frac{1}{M}\left(e^{-\frac{6 \pi \eta r}{M} t}+1\right) \times\left\{\frac{1}{m} \sum_{m} \frac{2 \pi n}{c} \alpha^{\prime} K_{m} \eta^{(1,2)} \sqrt{\frac{1}{\left(\Delta x_{m}\right)^{2}}+\frac{1}{\left(\Delta z_{m}\right)^{2}}}\right. \\
& \left.-\frac{1}{m} \sum_{m}\left[\left\{C_{a b s}+C_{\text {scatt }}\right\} \frac{n}{c} \sqrt{\frac{\varepsilon \varepsilon_{0}}{\mu \mu_{0}}} \sum_{i, j}\left\{\phi_{i j}^{(1)}(\mathbf{r})+\phi_{i j}^{(2)}(\mathbf{r})+2 \sqrt{\operatorname{tr}\left[W\left(\mathbf{r}_{1}, \mathbf{r}_{1}, 0\right)\right] \operatorname{tr}\left[W\left(\mathbf{r}_{2}, \mathbf{r}_{2}, 0\right)\right]} \times \eta_{i j}^{(1,2)} \cos \left[\left(\delta_{e}\right)_{m}\right]\right\}\right]\right\}
\end{aligned}
$$

In other words, the degree of mutual coherence affects the averaged velocity of the particle in the optical field.

To obtain a direct link between the averaged velocity of the test particles and the degree of coherence of the superposing fields, we should concentrate on some particular example of the test particles. Let the light scattering peculiar for those particles be described by the Rayleigh mechanism, whereas the scattering and the absorbing components of the optical force affecting them be much smaller than the gradient component. Then the averaged velocity of the nanoparticles in the optical field (or, more precisely, the velocity of trapping by the field) is determined by the degree of mutual coherence of the superposing fields:

$$
\bar{v}(t)=\frac{1}{M}\left(e^{-\frac{6 \pi \eta r}{M} t}+1\right) \frac{2 \pi n}{c} \alpha^{\prime} \eta^{(1,2)} \frac{1}{m} \sum_{m} K_{m} \sqrt{\frac{1}{\left(\Delta x_{m}\right)^{2}}+\frac{1}{\left(\Delta z_{m}\right)^{2}}} .
$$

Normalising the temporal changes in the averaged velocity to the maximum of trapping velocity for each corresponding moment of time, which would have been obtained for the case of absolutely coherent fields, we get

$$
\bar{v}_{r e l}(t)=\eta^{(1,2)} .
$$

Hence, the relative average velocity of the particles of nanometre-range sizes, which move in the optical field with spatially inhomogeneous energy resulted from interaction of partially coherent optical fields converging at the angle of $90^{\circ}$, makes estimating the degree of mutual coherence of these fields really possible.

\section{Results of simulation}

Using the methods suggested in the studies [18, 22, 25], one can illustrate the motion of particles of various sizes in the optical fields formed by superpositions of coherent or partially coherent beams. For receiving a more complete and vivid picture of the particle motion, it is also necessary to take into consideration a set of forces resulted from the liquid in which the particles are maintained in suspension. The behaviour of the test particles has been simulated without considering random Brownian forces which, as shown in the work [31], mask the information about the optical field and the corresponding coherence properties.

The optical field, in which we observe the motion of particles of different natures, is a result of the two plane waves with different degrees of coherence interacting in the interference geometry suggested above. Though a homogeneous intensity distribution is observed in the plane where the resulting field is detected, the spatial distribution of the Poynting vector can acquire inhomogeneous character and depend essentially upon the degree of coherence of the interacting beams.

Let us reproduce a full pattern of motion of the particle in the optical field. Essential polarisability of the golden particles and availability of absorption determine the following features of the motion: (i) spatial redistribution of particles among the regions of maxima and minima in the energy-inhomogeneous optical field, which is caused by action of the optical field, 
and (ii) further behaviour of the particles according to their sizes and the relations among the components of the optical force. The initial conditions determine a distribution of the particles over their velocities in the beginning of the motion (at the initial moments of time). Therefore the resulting optical force and the overall force that governs the motion of the particles are due to their location. The distributions of the average magnitudes of the Poynting vector presented in Movies 2, 3 and 4 have been simulated using Eq. (2).

Movie 2 and Fig. 2 illustrate the motion of $100 \mathrm{~nm}$ sized particles in the homogeneous vector optical field. Here the arrows indicate directions of the Poynting vector in the observation plane. The obtained distribution of the volume energy density is a result of incoherent interaction of the plane waves when there is no spatial modulation of polarization in the observation plane. As suggested by works $[15,16]$, then the modulation of the Poynting vector is absent too. The gradient component of the optical force caused by the gradient of the Poynting vector magnitude (i.e., the gradient of the volume energy density) does not change the direction of motion and the force gradient component is absent in this case.

The motion of the particle in our situation is governed by a resulting force caused by the scattering and absorbing components of the optical force, which is closely linked to the radiation pressure and the viscous friction in the liquid. The particle moves along the direction of energy current propagation in the detection plane. The velocity of the particle depends on the relationship between the field impulse and the viscous friction force.

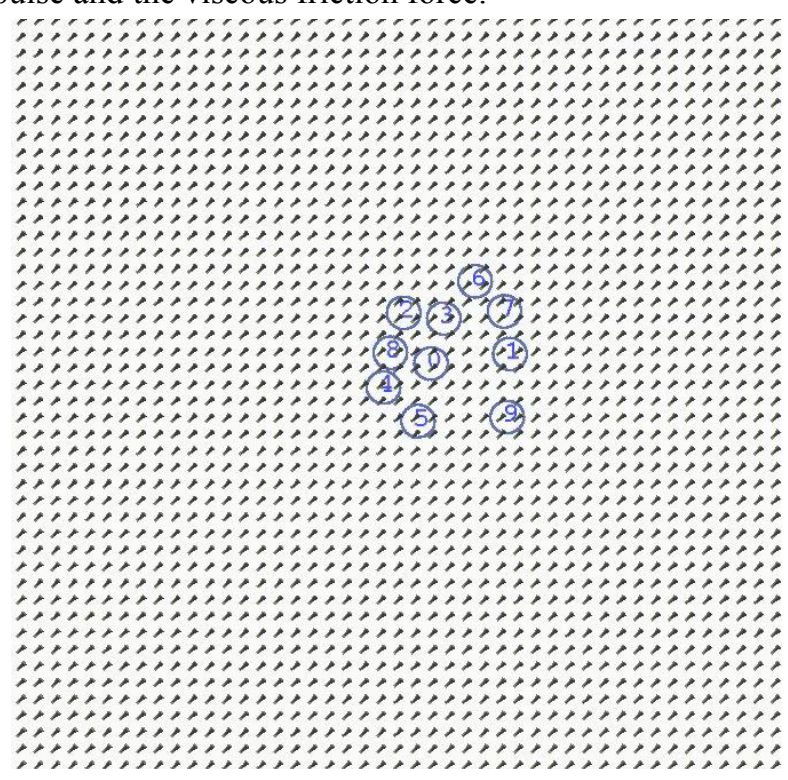

Fig. 2. Motion of particles with the sizes of about $100 \mathrm{~nm}$ in a homogeneous vector optical field. See also Movie 2 (format avi, $1.98 \mathrm{Mb}$ ).

Accounting for essential light absorption by the golden particles determines the direction of the resulting optical force. The absorption component becomes important for the particles of the size chosen by us and increasing size of the particle increases importance of that component. We can change the depth of energy inhomogeneity of the optical field by changing the degree of coherence of the interacting waves.

Movie 3 and Fig. 3 show the pattern of motion in the field formed by superposition of two partially coherent waves, with the degree of coherence 0.5 . Modulations of both the polarization 
and the volume energy density are seen in the observation plane, thus leading to the gradient component of the optical force. Since the gradient force is rather small when compared to the other two components of the optical force, its value is not enough to capture the particles. As a result, the particles move towards the region of potential energy minimum, according to the main laws of thermodynamics. The corresponding zones are localised in the region of Poynting vector minimum value.

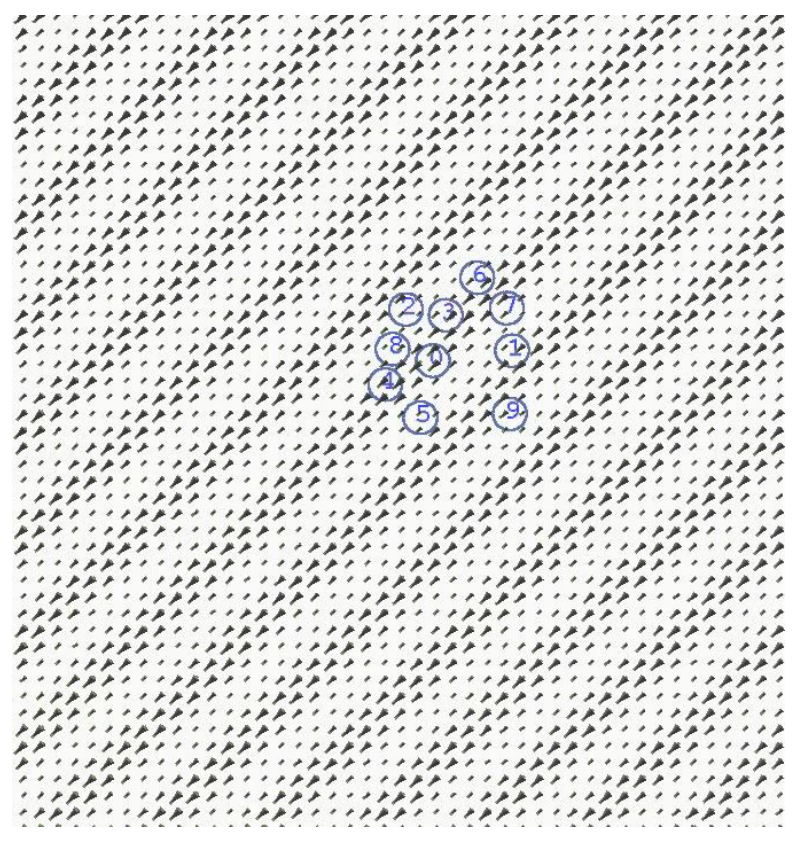

Fig. 3. Motion of particles in a field formed by superposition of two partially coherent waves characterised with the degree of coherence 0.5 . See also Movie 3 (format avi, 1.7 Mb).

The particles continue to move under the force, whose components are the scattering and absorbing components of the optical force and the viscous friction force. The results of our simulations fully justify the results of the experiment suggested in the work [25]. At the same time, the information about the gradient force associated with the gradient volume density and the degree of coherence of the superposing fields, is lost in the general pattern of particle motion.

Thus, in order to estimate quantitatively the degree of inhomogeneity of the optical field, one should choose the particles for which the gradient component of the optical force represents a determinative factor. Let us analyse the behaviour of particles with the sizes of about $1 \mathrm{~nm}$. According to the light scattering theory, it is precisely these particles that are considered as point ones and can be called as Rayleigh particles. As the simulation results demonstrate, the gradient force in this case gets much larger than the other components of the resulting optical force. That is why the field gradient can move the particles into the region of the maximum where trapping takes place (see Movie 4 and Fig. 4).

It is worthwhile that the optical field does not influence the particles whenever the homogeneous optical field results from incoherent interaction of the two superposing fields (i.e., the distribution gradient of the energy is absent).

Deeper modulation depths of the volume energy density correspond to higher degrees of coherence and, consequently, the degree of inhomogeneity of the optical field energy increases. Then the influence of the gradient component of the optical force increases, too (see Fig. 5). The 
resulting optical force has been calculated using Eq. (11). In the first approximation, the particles are considered to be motionless at the initial moment. Graphical representations of the optical force in Fig. 5 have been obtained after normalising the force at a certain moment of time to the magnitude obtained as if absolutely coherent fields interacted at the same moment.

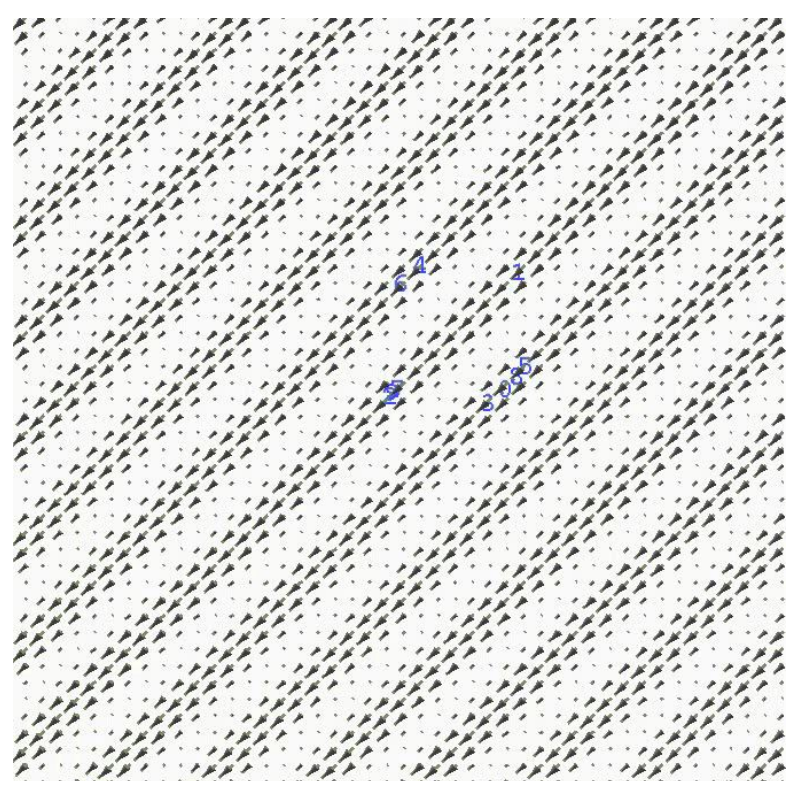

Fig. 4. Motion of particles with the sizes of about $1 \mathrm{~nm}$ in a vector optical field characterised with the maximal gradient. See also Movie 4 (format avi, $414 \mathrm{~kb}$ ).

Now the transverse component of the optical force is sufficient to trap nanoparticles and keep them in a 'capture' zone. Depending upon the initial positions of the nanoparticles, the velocities of their trapping by the field are different. These velocities are also affected by the volume energy density gradient.

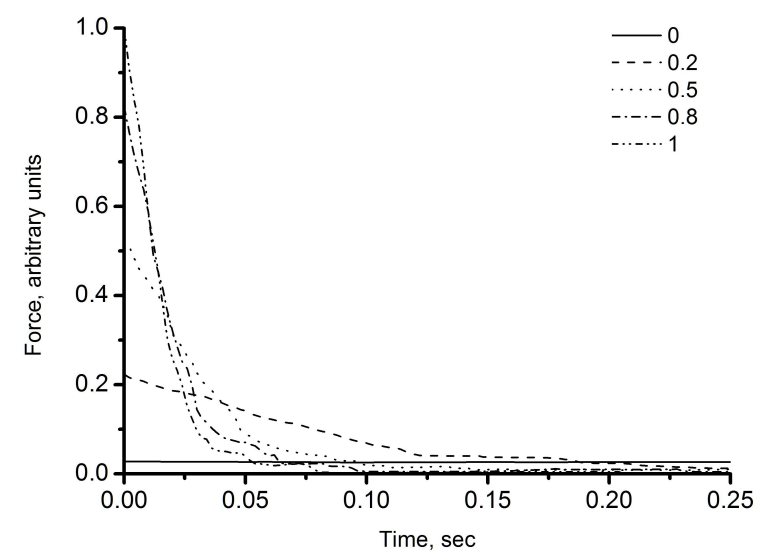

Fig. 5. Changes in optical force affecting the Rayleigh particles observed with time and changing degree $\eta^{(1,2)}$ of coherence of the interacting waves: the legend shows degrees of coherence that correspond to different curves.

The velocity of the nanoparticles depends on the spatial distribution of volume energy density in the observation plane and, accordingly, on the degree of coherence of the superposing waves. Changes in the degree of coherence cause changing velocity of redistribution of the particles in the optical field under the effect of optical forces that trap them (see Fig. 6). The 
velocity graphs presented in Fig. 6 are derived from Eq. (16). They describe normalised averaged velocities of the particles.

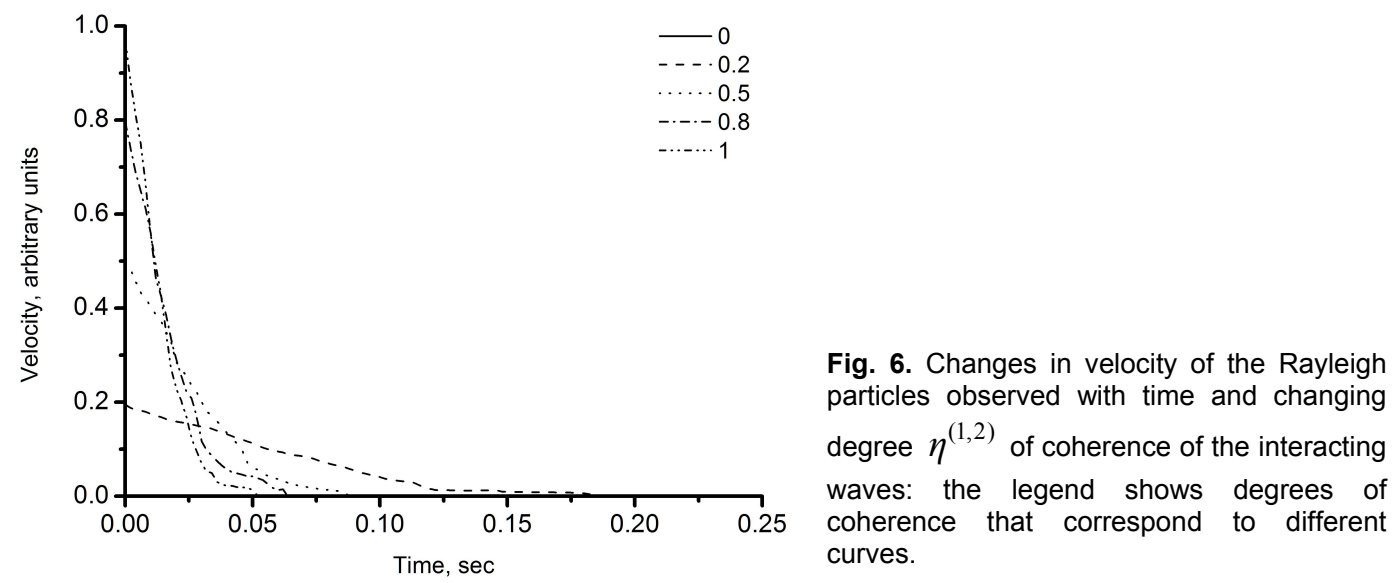

The maximal velocity and, correspondingly, the maximal resulting force are unequivocally related to the degree of coherence of the superposing initial beams, which form the optical field under study. For instance, the maximal velocity of the particles is 0.8 (see Fig. 3) provided that the degree of coherence is equal to 0.8 . Any changes in the degree of coherence of the superposing waves would change both the average velocity and the maximal velocity of particle 'trapping' in the maximal energy region of the field under analysis. For instance, the maximal velocity 0.5 of particle 'trapping' corresponds to the degree of coherence 0.5 .

As our computer simulation results testify, the particles are practically immediately 'trapped' inside the region of maximal gradient of the Poynting vector. As a result, the maximal velocity is achieved in the initial moments of time. At the same time, the nanoparticles possess some masses and so are characterised with some, to say, insignificant inertia. Because of this, a certain time, a period of 'relaxation' after 'trapping', is necessary for the particles to take their stable positions. This is attributed to (i) progressive decrease in the velocity after 'trapping' and (ii) the fact that the maximal velocity is chosen as an estimation criterion for the field imhomogeneity gradient and, consequently, for the degree of coherence.

Hence, the optical force, which causes trapping of the particles by the field, and the velocity of the latter are directly linked to the degree of coherence of the interacting optical fields. Then the velocity, being an easily measured quantity, may be chosen as an estimation parameter of the degree of coherence of the interacting fields.

\section{Conclusions}

In this paper we have suggested another instrument for estimating the degree of coherence of linearly polarised optical waves propagating along orthogonal directions. Namely, we have substantiated theoretically that the maximal normalised averaged velocity of a particle moving in the fields unambiguously corresponds to the degree of mutual coherence of the fields under analysis.

We have investigated the influence of degree of coherence of the mutually orthogonal waves , which are linearly polarised in the incidence plane, on the velocity of test nanoparticles. It has been shown that the spatial modulation of light polarization in the incidence plane causes spatial modulation of the volume energy density and changes in the gradient, scattering and the absorbing 
components of the resulting optical force that affects the test particles. As a consequence, the velocity of the nanosize particles can serve as a tool for determining the degree of coherence of the superposing waves.

\section{References}

1. Angel'skii O V, Ushenko A G, Arkhelyuk A D, Ermolenko S B, Burkovets D N and Ushenko Yu A, 2000. Laser polarimetry of pathological changes in biotissues. Opt. Spectrosc. 89: 973-978.

2. Ashkin A, 1970. Acceleration and trapping of particles by radiation pressure. Phys. Rev. Lett. 24: $156-159$.

3. Ashkin A, Dziedzic J M, Bjorkholm J E and Chu S, 1986. Observation of a single-beam gradient force trap for dielectric particles. Opt. Lett. 11: 288-290.

4. Chen C H, Tai P T and Hsieh W F, 2004. Bottle beam from a bare laser for single-beam trapping. Appl. Opt. 43: 6001-6006.

5. Garcés-Chávez V, Roskey D, Summers M D, Melville H, McGoin D, Wright E M and Dholakia K, 2004. Optical levitation in a Bessel light beam. Appl. Phys. Lett. 85: 4001-4003.

6. Ye J Y, Chang G, Norris T B, Tse C, Zohdy M J, Hollman K W, O'Donnell M and Baker J R, 2004. Trapping cavitation bubbles with a self-focused laser beam. Opt. Lett. 29: 2136-2138.

7. Okamoto K and Kawata S, 1999. Radiation force exerted on subwavelength particles near a nanoaperture. Phys. Rev. Lett. 83: 4534-4537.

8. Volpe C, Quidant R, Badenes G and Petrov D, 2006. Surface plasmon radiation forces. Phys. Rev. Lett. 96: 238101.

9. Angelsky O V, Besaha R N and Mokhun I I, 1997. Appearance of wave front dislocations under interference among beams with simple wave fronts. Opt. Appl. 27: 273-278.

10. Mandel L and Wolf E. Optical coherence and quantum optics. New York: Cambridge University Press (1995).

11. Zhao G and Lü B, 2005. Focal switching of partially polarized Gaussian Schell-model beams passing through a system with the aperture and astigmatic lens separated. J. Opt. A: Pure Appl. Opt. 7: 88-93.

12. Friberg A T, Visser T D, Wang W and Wolf E, 2001. Focal shifts of converging diffracted waves of any state of spatial coherence. Opt. Commun. 196: 1-7.

13. Wang Li-Gang, Zhao Cheng-Liang, Wang Li-Qin, Lu Xuan-Hui and Zhu Shi-Yao, 2007. Effect of coherence on radiation forces acting on a Rayleigh dielectric sphere. Opt. Lett. 32: 1393-1395.

14. Ashkin A, Optical trapping and manipulation of neutral particles using lasers. Singapore: Hackensack; New York: World Scientific (2006).

15. Bekshaev A Ya, Angelsky O V, Sviridova S V and Zenkova C Yu, 2011. Mechanical action of inhomogeneously polarized optical fields and detection of the internal energy flows. Adv. Opt. Technol. 2011: 723901.

16. Dienerowitz M, Mazilu M, Reece P J, Krauss T F and Dholakia K, 2008. Optical vortex trap for resonant confinement of metal nanoparticles. Opt. Express. 16: 4991-4998.

17. Malagninoa N, Pescea G, Sassoa A and Arimondob E, 2002. Measurements of trapping efficiency and stiffness in optical tweezer. Opt. Commun. 214: 15-24.

18. Zenkova C Yu, Gorsky M P, Soltys I V, Angelsky P O, 2012. On the possibilities of using inhomogeneity in light energy distribution for estimating the degree of coherence of 
superposing waves. Appl. Opt. 51: C38-C43.

19. Angelsky O V, Yermolenko S B, Zenkova C Yu and Angelskaya A O, 2008. Polarization manifestations of correlation (intrinsic coherence) of optical fields. Appl. Opt. 47: 5492-5499.

20. Angelsky O V, Zenkova C Yu, Gorsky M P and Gorodyns'ka N V, 2009. Feasibility of estimating the degree of coherence of waves at the near field. Appl. Opt. 48: 2784-2788.

21. Angelsky O V, Hanson S G, Zenkova C Yu, Gorsky M P and Gorodyns'ka N V, 2009. On polarization metrology (estimation) of the degree of coherence of optical waves. Opt. Express. 17: $15623-15634$.

22. Ellis J, Dogariu A, Ponomarenko S and Wolf E, 2005. Degree of polarization of statistically stationary electromagnetic fields. Opt. Commun. 248: 333-337.

23. Mujait M, Dogariu A, Wolf E, 2004. A law of interference of electromagnetic beams of any state of coherence and polarization and the Fresnel-Arago interference laws. J. Opt. Soc. Amer. A. 21: 2414-2417.

24. Zenkova C Yu, Gorsky M P, Maksimyak P P and Maksimyak A P, 2011. Optical currents in vector field, Appl. Opt. 50: 1105-1112.

25. Angelsky O V, Gorsky M P, Maksimyak P P, Maksimyak A P, Hanson S G and Zenkova $\mathrm{C} \mathrm{Yu}, 2011$. Investigation of optical currents in coherent and partially coherent vector fields. Opt. Express. 19: 660-672.

26. Angelsky O V, Bekshaev A Ya, Maksimyak P P, Maksimyak A P, Hanson S G and Zenkova $\mathrm{C} \mathrm{Yu}, 2012$. Orbital rotation without orbital angular momentum: mechanical action of the spin part of the internal energy flow in light beams. Opt. Express. 20: 3563-3571.

27. Angelsky O V, Bekshaev A Ya, Maksimyak P P, Maksimyak A P, Mokhun I I, Hanson S G, Zenkova C Yu and Tyurin A V, 2012. Circular motion of particles suspended in a Gaussian beam with circular polarization validates the spin part of the internal energy flow. Opt. Express. 20: 1351-11356.

28. Palik E D, Handbook of optical constants of solids. San Diego: Academic (1985).

29. Born M and Wolf E, Principles of optics. Oxford: Pergamon (1980).

30. Nevsky Yu A and Osiptsov A N, 2009. Simulating gravitational convection of the suspension. Lett. J. Theor. Phys. 35: 98-105.

31. Zenkova C Yu, Gorsky M P, Soltys I V and Angelsky P O, 2012. Influence of the Brownian movement on the motion of nano and micro-particles in the inhomogeneous optical field, Optoelectron. Rev. 20: 247-254.

Zenkova C. Yu., Gorsky M. P., Soltys I. V. and Angelsky P. O., 2012. Use of motion peculiarities of test particles for estimating degree of coherence of optical fields. Ukr.J.Phys.Opt. 13: $183-195$.

Анотація. У роботі обговорено взаємозв'язок між глибиною просторового розподілу енергії оптичного поля і швидкістю руху пробних частинок підчас їхньої взаємодії зі світлом за умов різних механізмів розсіяння світла. Запропоновано додатковий спосіб визначення ступеня когерентності хвиль, які інтерферують, поширюючись у взаємно ортогональних напрямках, $i$ ортогонально-лінійно поляризовані в площині падіння. Використання швидкості руху пробних частинок для очінки ступеня когерентності пропонується вперше. 